\title{
ALL-DERIVABLE POINTS OF NEST ALGEBRAS ON BANACH SPACES
}

\author{
WEISHUN XUE AND JinCHUAN HOU
}

\begin{abstract}
Let $\mathscr{N}$ be a nest on a real or complex Banach space $X$ and let Alg $\mathscr{N}$ be the associated nest algebra. $\Omega \in \operatorname{Alg} \mathscr{N}$ is called an additively all-derivable point if for any additive map $\delta: \operatorname{Alg} \mathscr{N} \rightarrow \operatorname{Alg} \mathscr{N}, \delta(A B)=\delta(A) B+A \delta(B)$ holds for any $A, B \in \operatorname{Alg} \mathscr{N}$ with $A B=\Omega$ implies that $\delta$ is an additive derivation. Assume that $P$ is an idempotent operator with range $\operatorname{ran}(P)=N_{0}$ for some nontrivial $N_{0} \in \mathscr{N}$. Let $\Omega \in \operatorname{Alg} \mathscr{N}$ be any operator satisfying that $P \Omega P=\Omega($ or $(I-P) \Omega(I-P)=\Omega)$. We show that, if $\left.\Omega\right|_{\operatorname{ran}(P)}\left(\right.$ or $\left.\left.\Omega\right|_{\operatorname{ran}(I-P)}\right)$ is injective or has dense range, then $\Omega$ is an additively all-derivable point. Moreover, if $X$ is infinite dimensional, then every additive map derivable at such an $\Omega$ is an inner derivation.
\end{abstract}

Mathematics subject classification (2010): 47L35, 47B47.

Keywords and phrases: Banach space nest algebras, derivations, all-derivable points.

\section{REFERENCES}

[1] R.-L. AN, J.-C. Hou, Characterizations of derivations on triangular rings: Additive maps derivable at idempotents, Linear Alg. Appl. 432 (2010), 1070-1080.

[2] E. Christensen, Derivations of nest algebras, Math. Ann. 229 (1977), 155-161.

[3] D.-G. Han, Continuity and linearity of nest algebras on Banach space, Chinese Ann. Math. 17B, 2 (1996), 227-236.

[4] M.-Y. JiAO, J.-C. Hou, Additive maps derivable or Jordan derivable at zero point on nest algebras, Linear Alg. Appl. 432 (2010), 2984-2994.

[5] X.-F. QI, J.-C. Hou, Characterizations of derivations of Banach space nest alegbras: All-derrivable points, Linear Alg. Appl. 432 (2010), 3183-3200.

[6] P. S Amuel, O. Zaris Ki, Commutative Algebra, Van Nostrand, New York, 1958.

[7] P. SEMrL, Additive derivations of some operator algebras, Illinois Journal of Math. 35 (1991), 234 240.

[8] L. Zhang, J. ZHU, J. WU, All-derivable point in nest algebras, Linear Alg. Appl. 433 (2010), 91-100.

[9] J. ZHU, Alle-derivable points of operator algebres, Linear Alg. Appl. 427 (2007), 1-5.

[10] J. ZHU, C. XIONG, All-derivable points in continuous nest algebras, J. Math. Anal. Appl. 340 (2008), $845-853$ 